\title{
Le sacrifice humain en Juda et Israël au premier millénaire avant notre ère
}

\author{
Thomas Römer/Lausanne
}

\section{Changements de paradigme}

Dans les recherches sur l'histoire et la religion de l'ancien Israël la question des sacrifices humains n'occupe qu'un rôle marginal. À la lecture de la Religionsgeschichte Israels in alttestamentlicher Zeit de R. Albertz, on a en effet l'impression que ce phénomène n'a jamais existé en Israël et en Juda, à l'exception de quelques dérapages syncrétistes dûs à l'influence assyrienne ${ }^{2}$. Cette vision quelque peu apologétique des choses fait apparaître un problème de sources. En cffet, la Bible juive, lincien Testament, est presque le seul document que nous possédons à ce sujet ${ }^{3}$. Les recherches exégétique et historique des dernières décennies ont apporté des modifications importantes concernant la datation et les milieux producteurs de la Bible. Il nous faut brièvement rendre compte de ces changements avant de pouvoir aborder la question des sacrifices humains. Dans les textes bibliques, ces rites, qui sont dans la plupart des cas des sacrifices d'enfants, sont en effet présentés comme une déviation du « vrai» culte yahwiste. Mais on sera attentif au fait que les deux parties de la Bible hébraïque qui évoquent ces types de sacrifice, le Pentateuque (Torah) et les livres historiques et prophétiques (Nebiim), sont d'abord le produit de l'idéologie des élites scribale et sacerdotale avant d'être le reflet des pratiques effectives de l'époque.

Depuis une dizaine d'années, il apparait de plus en plus que la Torah et les $\mathrm{Ne}$ biim sont fortement marqués par l'élaboration d'un yahwisme "orthodoxe» à l'époque perse ${ }^{t}$. Du coup, il devient fort probable que la présentation du dieu Yahré et de ses exigences rituelles et éthiques tient compte, au moins partiellement, des sensibilités théologiques des achéménides.

Par conséquent, une enquête sur la pratique des sacrifices humains en lsraël et Juda à partir des témoignages de l'Ancien Testament doit prendre en considération

'Lexpression «ancien Israël * est courante, mais peu précise. Du X' siècle jusqu'à 722 av. J.-C. - Israël s est un terme politique et désigne ce que les exégètes appellent souvent lc « Royaume du Nord . Il s agit d'un royaume relativement important en Sỵrie-Palestinc dont le petit royaume de Juda est. à pant quelques interruptions. le vassal. A près l'incorporation d'Israël dans l'empire assyrien. Judu revendique le titre d'Israël. À partir de loccupation babylonienne «/sraël" devient de plus en plus un terme idéologique désignant la comınunauté des vénérateurs de Yahvé.

- R. Albertz. Religionsgeschichte Isruels in alttestamentlicher Zeit. 2 "ol., Grundrisse zum Altcu Testament 8. (Cöttingen 1992) 297-303.

${ }^{3}$ Larchéologie palestinienue ne permet guère davancer dans la question des sucrifices humains.

+ Cr. par exemple. E.T. Mullen Jr. Ethinic Mylls and Pentateuchal Foundations. A New Approach to the formation of the Pentateuch, Semeia Studies, (Atlanta 1997). 
le: fait que nous n'avons pas d'accès immédiat à des documents anciens, même s'il est clair que les scribes de l'époque perse se sont servis de matériaux datant de l'époque des monarchies judéenne et israélite. L'enquête «socio-archéologique» de Jamieson-Drakes suggère que les documents les plus anciens que nous puissions reconstruire datent de la fin du VIII" siècle avant notre ère.

Le deuxième changement de paradigme concerne le dossier du monothéisrne juif. Même si l'hypothèse d'un «monothéisme primitif » en Israël n'a pas été entièrement abandonnée, on ne peut nier aujourd'hui l'évidence d'une élaboration tardive de l'idéologie monothéiste. Ce n'est qu'à l'époque perse que Yahvé devient le Dieu unique et les autres divinités des idoles ${ }^{6}$. Jusqu'au VIe siècle avant notre ère. la religion judéenne, dans ses manifestations officielles comme dans les pratiques dites «populaires », était polythéiste et dans certains milieux le yahwisme polythéiste s'est longtemps perpétué . À l'époque royale, Yahvé était vénéré comme Dieu national, accompagné d'une déesse, Ashéra, et entouré d'autres divinités, autochtones ou importées. Si les textes bibliques parlent dans la plupart des cas des sacrifices humains d'une manière critique, cela ne signifie nullement qu'ils ne faisaient pas partie du culte yahwiste officiel jusqu'à l'époque perse. De plus, il y a un type de sacrifice humain qui n'est jamais critiqué dans les textes bibliques: le herem. C'est par ce rituel que nous commencerons notre enquête avant d'aborder le problème des sacrifices d'enfants: l'offrande des premiers-nés et le rituel du "passage par le feu ". À la fin nous esquisserons brièvement l'évolution de l'idéologie sacrificielle dans l'Israël ancien qui aboutit au rejet des sacrifices humains à l'époque perse.

\section{Le herem - un sacrifice de reconnaissance}

Comme dans tout le Proche Orient ancien, la guerre est un événement sacral en Israël et en Juda. C'est le Dieu national qui mène la bataille pour son peuple, et c'est donc à lui que revient la victoire, si victoire il y a. Par conséquent, tout le butin, vivant et matériel, lui revient de droit et doit être brûlé. Cette pratique, qui n'est pas limitée au Proche-Orient ancien ${ }^{8}$, était désignée en Juda et en Israël par le terme de $h_{\text {herem }}{ }^{9}$ et son destinataire était Yahvé. Ainsi 1 S 15,3 stipule:

«Vous devrez pratiquer totalement le herem... Tu mettras tout à mort, hommes et femmes, enfants et nourrissons, bœufs et moutons, chameaux et ânes».

Le terme herem provient d'une racine signifiant "consacrer». " mettre à part », et peut être traduit par «tabou» ${ }^{10}$. Dans le contexte de la guerre sacrale, le substantif

${ }^{5}$ D.W. Jamieson-Drake, Scribes and Schools in Monarchic Judah. A Socio-Archeological Approach, JSOT.S 109, (Sheffield 1991).

${ }^{6} \mathrm{~F}$. Stolz, Einführung in den biblischen Monotheismus, Die Theologie, (Darmstadt 1996); T. Römer, "L'exil à Babylone, creuset du monothéisme», MdB 110 (1998) 43-45. 47-49.

${ }^{7} \mathrm{La}$ meilleure attestation que nous possédons est le cas de la colonie juive d'Eléphantine, vénérant (à l'égyptienne?) une triade divine.

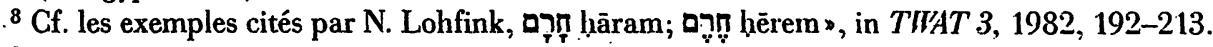

${ }^{9}$ A ce sujet cf. C. Brekelmans, Det herem in het Oude Testament, (Nijmegen 1959) et P.D. Stern, The Biblical Herem: A Window on Israel's Religious Experience, Brown Judaic Studies 211, (Atlanta, GA 1991).

${ }^{10}$ M. Malul, "Taboo », in K. van der Toorn, B. Becking et P.W. van der Horst (éd.) Dictionary of Deities and Demons in the Bible, (Leiden et al. 1995) cols. 1559-1565. 
devient un terme technique pour la rémission du butin à la divinité. On rencontre également cette expression chez les voisins immédiats d'Israël, les Moabites. Dans l'inscription du roi moabite Mésha (vers 850 avant notre ère), l'aspect sacrificiel du herem est clairement attesté ${ }^{11}$ :

" Je combattais contre la cité [= Atarot] et je la pris. Et je tuai tout le peuple. La ville devint offrande pour Kamosh de la part de Moab...» Kamosh me dit: ‘Va, prends Nébo sur Israël ’... Je la pris et je tuai tout, sept mille hommes libres et étrangers, femmes libres et étrangères, et concubines. J'en avais fait un herem pour Ashtar-Kamosh» (lignes 11-17).

Les sacrifices humains dans le cadre d'une victoire militaire étaient pratiqués en Israël et Juda dans la première moitié du premier millénaire av. J.-C. S. Niditch constate que "the ban-as-sacrifice tradition is an on-going thread in ancient Israelite tradition "12. Dans le cadre du sacrifice du herem les victimes appartiennent à un groupe ennemi et sont exterminées (dans la plupart des cas brûlées) en même temps que des animaux et du butin inanimé. Le sacrifice humain s'inscrit ici dans une logique sacrificielle plus globale.

Il est difficile à imaginer que le herem ait été systématiquement appliqué, puisqu'il est contraire aux intérêts économiques liés à la guerre dans l'Antiquité. Certains rois assyriens se vantent en effet d'avoir entièrement exterminés leurs ennemis $^{13}$, ce qui ne les empêche nullement de faire figurer sur des bas-reliefs des rangées impressionnantes de prisonniers et de butin ${ }^{14}$. De même, le texte de 1R 9,20-21, qui décrit le règne de Salomon avec des traits empruntés aux souverains assyriens ${ }^{15}$, constate que Salomon tire profit des ennemis soustraits au herem en les astreignant à la corvée servile ${ }^{16}$. La loi du herem est avant tout une construction idéologique affirmant que tout butin est propriété de la divinité. Cela n'exclut nullement que le herem soit occasionnellement réalisé, peut-être en offrant un ou plusieurs sacrifices comme pars pro toto ${ }^{1 ?}$.

"Récemment R.D. Nelson, «herem and the Deuteronomic Social Conscience», in M. Vervenne et J. Lust (éd.). Deuteronomy and Deuteronomic Literature. Festschrift C.H.II' Brekelmans, BEThL CXXXIII (Leuven. 1997) 39-54, a contesté l'interprétation du herem comme sacrifice. Il propose de le voir comme simple destruction du butin. et fait remarquer que, dans la pratique du herem à la suite d'une guerre. il n'est jamais fait mention ni d'un autel ni d'un prêtre. Ces arguments ne sont guère concluants. d'autant moins que le roi est le médiateur par excellence et qu'il est habilité à sacrifier ou à délequer les sacrifices. Notons encore que $\mathrm{Dt} 13,7$ évoque également un contexte sacrificiel.

12 S. Niditch, Ilar in the Hebreu Bible. A Study in the Ethics of Violence, (New York - Oxford 1993) +6.

${ }^{13}$ Cf. par ex. les annales d'Assur-nasir-pal, citées par K.L. Younger Jr, Ancient Conques! Accounts. A Study in Ancient Near Eastern and Biblical History Writing, JSOT.S 98, (Sheffield 1990) 236.

it Line contradiction similaire se trouve dans la stèle de Mésha, lequel, après avoir appliqué le ḥerem contre Israël, a néanmoins des prisonniers israélites à sa disposition.

15. La première version du règne de Salomon (1R 1-11) a été sans doute rédigée lors de l'époque assigrienne. cf. E.A. Knauf, Die L'muelt des Alten Testamenls, NSK AT 29, (Stuttgart 1994) 115.

is 11. Weinfeld, The Ban on the Canaanires in the Biblical Codes and its Historical Development $x$. in A. Lemaire et B. Otzen (éd.), History and Traditions of Early Israel, SVT 50 (Leiden 1993) 1+2-160. conclut à partir de ce texte que le herem * was never carried out • (159).

1? Cette pratque est peut-être refletée en $1 \mathrm{R} 20$, où c est seulement le roi étranger qui a été désigné comme herem (cf. v. +2 ). 
Contrairement aux autres types de sacrifices humains, le herem nest ni condamné ni critiqué par les autcurs bibliques. Ceci s'explique par le fait que le Ierme devient dès le VI" siècle avant notre ère un concept clé de l'orthodoxie deutéronomiste, laquelle construit une théorie de l'extermination totale des habitants autochtones de C'anaan visant en réalité à l'époque perse la construction d'une communauté ethnico-religieuse hasée sur la séparation stricte d'avec les autres peuples ${ }^{18}$. C'est d'ailleurs à partir de cette utilisation exclusivement idéologique que les rabbins ont interprété le herem dans le sens de l'excommunication des infidèles.

\section{Le sacrifice des premiers-nés}

L'idée que les prémices d'une récolte ou le premier-né d'un animal (domestique) sont à offrir à la divinité est largement répandue dans de nombreuses religions. Ce sacrifice sert soit à garantir la fertilité du champ ou du troupeau, soit à reconnaître symboliquement la divinité comme auteur et propriétaire des récoltes et des naissances ${ }^{19}$. Dans la Bible hébraïque, le sacrifice des premiers-nés concerne également les enfants mâles. Ainsi la législation du "Code de l'Alliance» stipule:

«Tu me donneras le premier-né de tes fils. Tu feras de même pour ton bœuf et pour tes moutons: il restera sept jours avec sa mère; le huitième jour, tu me le donneras» (Ex 22,28-29; cf. Ex 13,2).

D'autres textes reflètent la même exigence, mais prévoient la substitution du sacrifice humain par un sacrifice animalier:

"Tu feras passer à Yahvé tout ce qui ouvre le sein maternel... Tout premier-né d'homme parmi tes fils, tu le rachèteras" (Ex 13,11-12, cf. Ex 34,19-20).

Souvent on a conclu que la formulation qui ne mentionne pas le "rachat " appartiendrait à une époque fort archaïque. On aurait alors très vite remplacé le sacrifice du premier-né humain par une victime animalière. Or, les textes bibliques montrent qu'il n'en est rien. D'une part, il est loin d'être acquis que le texte d'Ex 22 remonte à une époque très ancienne ${ }^{20}$; d'autre part, plusieurs textes montrent que les sacrifices des premiers-nés humains furent pratiqués en Juda durant la monarchie et encore au début de l'époque perse.

Une texte du livre de Michée contient une parodie critique du culte sacrificiel ${ }^{21}$ :

"Yahvé voudra-t-il des milliers de béliers? des quantités de torrents d'huile?

18 Il est communément adınis que des textes comme Dt 7 sont totalenıent fictifs, cf. G. Braulik, "Die Völkervernichtung und die Rückkehr Israels ins Verheißungsland. Hermeneutische Bemerkıungen zum Buch Deuteronomiuın », in M. Vervenne et J. Lust (éd.), op. cit. (n. 11) 3-38.

${ }^{10}$ Cf. H. Gese, "Ezechiel 20,25f. und die Erstgeburtsopfer", in H. Donner, R. Hanhart et R. Smend (éd.), Beiträge zur alttestamentlichen Theologie. Festschrift für W'alther Zimmerli zum 70. Geburtstag (Göttingen 1977) 140-151; p.'147.

${ }^{20} \mathrm{Cf}$. O. Kaiser, «Den Erstgeborenen deiner Söhne sollst du mir geben. Erwägungen zum Kinderopfer im Alten Testament », in O. Kaiser (éd.), Denkender Glaube: Festschrift Carl Heinz Ratschow, (Berlin - New York 1976) 24-48; G.C. Heider, The Cult of Molek. A Reassessment. JSOT.S 43, (Sheffield 1985) 253 et surtout J. Van Seters, * The Law on Child Sacrifice in Exod. 22: 28b-29 (à paraître dans ETL). Je remercie John Van Seters d'avoir mis à ma disposition cet article très stimulant.

${ }^{21}$ La datation de ce texte ne fait pas l'unanimité. Selon J. Van Seters, loc. cit. (n. 20), Mi (j,1-8 daterait au plus tôt de l'époque exilique. 
Donnerai-je mon premier-né pour prix de ma révolte? Et l'enfant de ma propre chair pour mon propre péché?» (Mi 6,7).

Cette critique prophétique dénonce la dissociation des sacrifices de la justice sociale (cf. v. 8). Mais cette dénonciation montre du coup que les sacrifices des premiers-nés humains font partie du culte yahwiste officiel. Le fait que le sacrifice humain soit mentionné en dernier indique, selon Mosca, qu'il a été considéré comme le sacrifice le plus précieux ${ }^{22}$. Il n'y donc pas d'évolution d'un sacrifice humain pratiqué vers sa substitution. Les deux possibilités coexistent, comme c'est également le cas dans la colonie phénicienne de Carthage. Dans le cimetière d'enfants de Salammbô on a trouvé pour le VIIe siècle des ossements d'enfants et des ossements animaliers, "preuve manifeste de l'existence des sacrifices de substitution "23. Le sacrifice des premiers-nés humains en Judah n'a sans doute jamais été conçu sans la possibilité d'un rachat, mais il a été aussi pratiqué comme le plus grand sacrifice pour Yahvé2+, et ceci jusqu'aux débuts de l'époque perse. Un texte du livre d'Ezéchiel datant de cette époque contient un énoncé tout à fait singulier. Dans un discours prophétique Yahvé s'exprime au sujet des premiers-nés:

«Je leur donnais moi-même des lois qui n'étaient pas bonnes et des coutumes qui ne font pas vivre. Je les souillais par leurs offrandes: les sacrifices de tous les premiers-nés» (Ez 20,25-26).

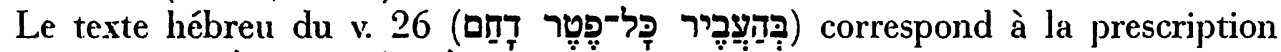

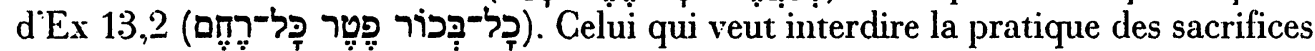
des premiers-nés ne peut donc les déclarer comme idolâtres ${ }^{25}$. Ils sont trop fortement enracinés dans le culte de Yahvé. Ainsi l'auteur, appartenant à la nouvelle orthodoxie, se voit obligé d'aroir recours à un subterfuge audacieux, prétendant que Ýahvé lui-même aurait pronulgué des mauvaises lois en décrétant les sacrifices humains (cf. également v. 21).

Selon certains textes bibliques des sacrifices d'enfants auraient été pratiqués à Jérusalem. dans un endroit appelé Tophet (ou Taphet) ${ }^{26}$. Ce mot, dont la signification exacte n est pas connue ${ }^{27}$, est également utilisé par les spécialistes pour désigner les emplacements que les colonies phéniciennes avaient réservés au sacrifice,

22: P.C. Mosca. Child Sacrifice in Canaanite and Israelite Religion: A Study in Mulk and mlk, Ph.D. dissenation. non publiée. (Harvard 1975) 225. L'avis opposé de R. de Vaux, Les sacrifices de lincien Testament. (Paris 196+) 69-70 ne tient pas compte du verset suivant et ine semble assez apologétique.

${ }^{2}$ E. l.ipiński, aSacrifices denfants à Carthage et dans le monde sémitique oriental», in E. Lipiński .éd.). Studia Phoenicia 1\%. Carthago. Orientalia Lovaniensia Analecta 26 (Leuven 1988) 151-185: citation p. 151.

$2+$ J.D. Levenson. The Death and the Resurrection of the Beloved Son. The Trunsformation of Child Sacrifice in Judaism and Christiunity; (New Haven - London 1993) parle d'un a theological ideal about the sperial place of the first-born son, an ideal whose realization could range from literal to non-literal implementation. that is from sacrifice to redemption * (p. 9).

2: Selon une thèse de John Van Seters (cf. n. 20), la loi du sacrifice du premier-né humain s"iusscrirait dans unc tradition sacerdotale.

20 Cf. notamment 2R 23.10 et Jr 7.31-32.

2: J. Day. Holech. A Cod of Human Sarrifice in the Old Testanent. Lniversity of Cambridge: Oriental Publications +1 (Cambridge et al. 1989) 2t-31 envisage un lien avec le mol aramirumtapiö (átre. foyers). La vocalisation de tophet est sans doute artificielle ì partir du mot boshet (ahontes). 
ou du moins à l'enterrement de jeunes enfants ${ }^{28}$. Le Tophet judéen ne peut guère être mis en rapport avec les sacrifices des premiers-nés, puisque les textes en lien avec ce lieu, c'est-à-dire la vallée de Hinnom, évoquent le passage par le feu de filles et de garçons, sans préciser leur âge. De plus, $2 \mathrm{R}$ 23,10 décrit ce sacrifice comme offert à «Molek». De nombreux chercheurs interprètent ce nom comme celui d'une divinité distincte de Yahvé, et concluent que la plupart des sacrifices humaines étaient pratiqués dans le cadre d'un culte non-yahwiste.

\section{Les sacrifices de «Molek»}

Quatre textes bibliques mentionnent le mot «Molek» en lien avec des sacrifices d'enfants ${ }^{29}$. Traditionnellement, on a vu dans Molek une divinité sanguinaire, friande d'holocaustes humains. Or, $\mathrm{O}$. Eissfeldt avait rapproché molek du mot punique molk, qui désigne simplement selon lui un type de sacrifice (non nécessairement humain) ${ }^{30}$. Cette théorie a connu un grand succès ${ }^{31}$, mais G.C. Heider et J.-Day ont récemment à nouveau interprété Molek comme le nom déformé d'une divinitéb $^{32}$. Selon eux, il s'agirait de la divinité chthonienne Maliku, attestée dans des textes ougaritiques ${ }^{33}$. D'autres identifications ont été également proposées: Mil$\mathrm{kom}^{34}, \mathrm{Baal}^{35}$ et Adad(-Milki) ${ }^{36}$. Aucune de ces solutions n'est satisfaisante.

Une divinité Maliku existe apparemment à Ougarit, mais ses attestations sont très peu nombreuses ${ }^{37}$, et n'ont aucun lien avec des sacrifices humains ${ }^{38}$. Lidentification de Molek à Milkom ne peut se baser que sur 1R 11,7, mais la mention de Molek dans ce texte est due à une erreur de scribe. Jr 32,5 évoque Baal et Molek dans un même contexte, mais il s'agit de deux divinités distinctes ${ }^{39}$. Le dossier en

28 Nous n'allons pas discuter la question de savoir si les Phéniciens d'outre-mer ont pratiqué des sacrifices d'enfants. Malgré les réserves de M. Gras, P. Rouillard, et J. Teixidor, L'univers phénicien (Paris 1989) 176-180 il me semble difficile d'interpréter les différentes inscriptions dans le sens d'un enterrement d'un mort-né ou d'un nourrison mort de mort naturelle, cf. également S. Brown, Late Carthaginian Child Sacrifice, JSOT/ASOR Monograph Series 3 (Sheffield 1991). Cf. l'article de C. Grottanelli, ci-dessous p. 52-58.

${ }^{29} \mathrm{Lv} 18,21 ; 20,2-5 ; 2 \mathrm{R} 23,10 ; \mathrm{Jr} 32,5$. Molek apparaît encore en $1 \mathrm{R} 11,7$, mais là il s'agit d'une erreur de scribes pour Milkom, le dieu des Ammonites.

30 O. Eissfeldt, Molk als Opferbegriff im Punischen und Hebräischen und das Ende des Gottes Moloch, Beiträge zur Religionsgeschichte des Altertums 3 (Halle/Saale 1935). Eissfeldt postule une racine $y / h-1-k$ qui dans la forme causative signifierait « ce qu'on apporte (comme offrande) $\triangleright$.

${ }^{31}$ Cf. l'histoire de la recherche détaillée chez G.C. Heider, The Cult of Molek. A Reassessment, JSOT.S 43, (Sheffield 1985).

32 Vocalisé selon le mot boshet (cf. n. 27)

${ }^{33}$ Op. cit. (n. 27 et 29).

${ }^{3+}$ M. Gras et al., op. cit. (n. 28), 173.

35 D. Edelman, «Biblical Molek Reassessed», JAOS (107, 1987) 727-731.

${ }^{36}$ R. Albertz, op. cit. (n. 2), 300-301.

37 À notre connaissance il s'agit de KTU 1.100.41; 1:107.17 et RS 1986/2235.17.

${ }^{38}$ Sans parler de la difficulté d'éclairer des textes bibliques des VIle-Vé siècles avec des textes du XIII' siècle.

${ }^{39}$ Cf. J. Day, op. cit. (n. 27) 34-36. Jr 19,5 parle de la construction des «hauts lieux » pour le Baal et évoque en même temps des sácrifices d'enfants. Il s'agit d'un texte tardif, midrashique, «an erroneous exegesis of 19.4» qui essaie d'attribuer les sacrifices humains de 7,31 a Baal, cf. 
faveur d'Adad-Milki est également très faible. $2 \mathrm{R}$ 17,31 parle d'une population "étrangère » ${ }^{40}$; de plus l'identification d'Adrammelek avec Adad n'est nullement sûr ${ }^{+1}$.

Cette difficulté à cerner l'identité du dieu Molek pourrait parler en faveur de la thèse selon laquelle molk désignerait un certain type de sacrifice. Smelik a modifié la thèse d'Eissfeldt de la manière suivante: molk aurait été à l'origine un terme sacrificiel désignant des sacrifices d'enfants offerts à Yahvé. À l'époque perse, des scribes auraient délibérément changé molk en molek pour en faire une idole, et pour faire des sacrifices d'enfants un culte idolâtre ${ }^{42}$. La thèse est séduisante. Reste la difficulté d'expliquer l'arrivée d'un mot punique en Palestine ${ }^{43}$; et d'ailleurs Lv 20,5 s'oppose à la compréhension de molek comme désignant d'un type d'offrande.

Il ne reste qu'une solution: molek était à l'origine melek, et constituait un titre pour Yahvé. Le mot melek est souvent employé dans l'Ancien Testament pour Yahvé (plus que 50 fois). On connaît plusieurs appellations pour Yahvé, comme Yahvé Sebaot ou Yahvé Shomron. Il est donc possible que les sacrifices d'enfants lui furent offerts en tant que Yahvé-Melek. Certains textes font d'ailleurs apparaître un contexte royal du passage des enfants par le feu ${ }^{4+}$. Notre thèse trouve une confirmation dans la traduction grecque de molek en Lv. J. Lust a démontré que le traducteur grec a lu en Lv 18,21 et 20,2-5 melek et l'a interprété comme titre pour $Y^{\prime}$ ahvét ${ }^{45}$. Les critiques prophétique et sacerdotale de l'époque perse confirment le fait que les sacrifices de «Molek» étaient offerts à Yahvé:

Lv 18,21: «Tu ne livreras pas l'un de tes enfants pour le faire passer à Me/olek et tu ne profaneras pas le nom de ton Dieu". Selon ce texte un sacrifice d'enfants à Molek est une profanation du nom de Yahvé, ce qui ne fait sens à condition que meTek puisse être accolé au nom du dieu d'Israël. Jr 7,31 va dans le même sens: "Ils construisent les hauts-lieux du Tophet ... pour brûler leurs fils et leurs filles par le feu, ce que je nai pas commandé et ce qui me n'est jamais venu à l'esprit». L'auteur affirme que Yahvé n'a jamais ordonné des sacrifices d'enfants, ce qui signifie que pour ses adversaires Yahvé avait bel et bien exigé de tels sacrifices ${ }^{+6}$.

II. McKane, A Critical and Exegetical Commentary on Jeremiah. Volume I, ICC, (Edinburgh 1986) 45.5. La précision «à Baal > manque d'ailleurs dans la version grecque.

to Il sagit d'un texte hautement polémique de l'époque postexilique; pour plus de détails cf. J.-D. Macchi, Les Samaritains. Histoire d'une légende. Israël et la province de Samarie, Le Monde de la Bible 30. (Genève 1994) 158-161.

${ }^{+1}$ J. Ebach et U. Rüterswörden, ADRMLK, Moloch> und BA'AL ADR. Eine Notiz zum Problem der Moloch-Verehrung im alten Israel s UF 11 (1979) 219-226.

+2 K.A.D. Smelik, Moloch, Molekh OR Molk-Sacrifice? A Reassessment of the Evidence Concerning the Hebrew Term Molekh > SJOT 9/1 (1995) 133-142. R. de Vaux, op. cit. (n. 22) 79-81 avait déjà émis une hypothèse allant dans le même sens. Selon lui les scribes n'auraient plus connu le vrai sens du mot molk.

${ }^{43}$ Le mot molk n'est apparemment pas attesté en Phénicie.

+4 2R 3//2 Ch 28.3; 2R 21,6//2 Ch 33,6.

4.5 J. Lust, Molek and APXON, in E. Lipiński (éd.), Studia Phoenici XX. Phoenicia and the Bible, Orientalia Lovanensia Analecta 44, (Leuven 1991) 193-208. Dans sa conclusion il écrit: "The: translator of Leviticus ... vocalized the tern as melek sking, and probably understood it as a tille of ITHH * (208).

*) Larguneut est en quelque sorte comparable au cas d'Ez 20,25s. 
(Ce sacrifice pour Yahvé-Melek s'effectuait par combustion ${ }^{+?}$. Il apparait selon nos textes au VIII" siècle, et perdure au moins jusqu'à l'époque babylonienne ${ }^{+8}$. Quel est son but ? Lipiński pense qu'il s'agit d'un contrôle rituel de naissances permettant de supprimer, via un sacrifice, des enfants non souhaités ou nés avec des handicaps physiques ou psychiques. En effet, Jérusalem connaît à la fin du VIIr siècle. lorsque ces sacrifices surgissent, une explosion démographique ce qui expliquerait la volonté de reduire les naissances ${ }^{\dagger 9}$. Cette hypothèse nous paraît cependant trop "moderne»; clle est de plus contredite par la conception israélite du sacrifice, qui interdit de sacrifier à la divinité tout être ayant un défaut.

À notre avis, les sacrifices par le feu s'expliquent mieux à partir d'un récit mettant en scène un sacrifice humain par le roi moabite Mésha:

"Quand le roi Moab vit que la bataille était perdue pour lui... il prit son fils premier-né qui devait régner à sa place, et l'offrit en holocauste sur la muraille. Il ỵ eut un grand courroux sur Israël. Ils décampèrent de chez lui et retournèrent au paỵs ” (2 R 3,26-27).

C'est dans un contexte de crise militaire que Mésha ne voit plus d'autre solution que d'offrir ce qu'il a de plus cher: son fils, successeur au trône. Le texte ne dit pas à quelle divinité cet holocauste était destiné: à Kamosh ou à Yahvé. Ce récit, qui a surmonté la censure des rédacteurs bibliques ${ }^{50}$, permet d'expliquer le passage des enfants (qui ne sont pas forcément des nourrissons) par le feu comme un sacrifice de dernier recours lors de crises graves. Les attestations bibliques corroborent cette interprétation. Le sacrifice d'Akhaz $(2 \mathrm{R} 16,3)$ se situe dans le contexte de la guerre syro-éphraïmite, celui de Manassé $(2 \mathrm{R} 21,6)$ au moment de l'occupation assrrienne. 2R 17,17 relate des sacrifices d'enfants juste avant la chute de Samarie. et $\mathrm{Jr} 7,31-32 ; 32,35$ pourraient refléter le désarroi de la population au moment du siège de Jérusalem.

Contrairement aux offrandes des premiers-nés dont la pratique était (théoriquement) régulière, les sacrifices recourant au "passage par le feu» constituent par conséquent des rituels destinés à invoquer l'intervention de la divinité à l'occasion de situations de grand danger.

\section{Les sacrifices humains à l'époque perse: polémique et fin}

Nous avons déjà souligné en introduction l'importance qu'occupe l'époque perse dans l'élaboration d'un monothéisme yahwiste par l'intelligentsia sacerdotale et laïque. La mise en place progressive de ce nouveau système religieux (dont le judaïsme est l'héritier direct) s'accompagnera de l'exclusion des sacrifices humains.

${ }^{+7}$ On trouve deux expressions «brûler» et « faire passer par le feu». Ce parallélisme contredit la thèse de M. Weinfeld, "The Worship of Molech and of the Queen of Heaven and its Background" $U F+1$ (1972) 133-154, qui y voit un rite de dédicace symbolique.

+3 Selon 2 R 23,10 Josias aurait éradiqué cette pratique, mais le livre de Jr atteste sa survivance après le siège de Jérusalem par les Babyloniens.

+" E. Lipiński, loc. cit. (n. 23), 159-161. Lipiński s'inspire des thèses de Stager sur les sacrifices d'enfants puniques, cf. L.E. Stager et S.R. Wolff, * Child Sacrifice at Carthage - Religious Rite or Population Control? », BAR 10/1 (1984) 31-51.

${ }^{50}$ Peut-être sont-ils toutefois responsables de la suppression du nom de la divinité. 
On peut spéculer sur une éventuelle influence mazdéenne dans le bannissement de ces sacrifices, bien que celle-ci demeure difficile à établir ${ }^{51}$. Mais de toute façon, la nouvelle orthodoxie juive promulguant la vénération de Yahvé comme Dieu unique et transcendant, que les Perses pouvaient sans autre identifier à Ahura-Mazda, devait nécessairement abolir la pratique des sacrifices humains que certains voulaient apparemment perpétuer'52. De simples interdits (cf. Dt 18,10; Lv 18,21) ne suffisaient pas; l'idée selon laquelle Yahvé lui-même avait demandé des sacrifices d'enfants était trop répandue. Pour cette raison Ez 20,25 assimile le sacrifice des premiers-nés humains à un mauvais commandement que Yahvé aurait donné dans sa colère $^{533}$. Le récit du sacrifice (substitué) d'Isaac en Gn 22 s'inscrit dans une stratégie similaire ${ }^{5 t}$. Dans ce texte, Dieu (Elohim) demande à Abraham de lui sacrifier son fils, alors qu'un ange de Yahvé le retient au dernier moment, ouvrant ainsi la voie à une substitution animalière. Il est hautement significatif que Gn 22 soit le seul récit de la geste d'Abraham dans lequel le patriarche offre un sacrifice. Certes, tout au long de ses migrations, il construit des autels, mais il les utilise exclusivement pour "invoquer le nom de Yahvé». On a presque l'impression que les auteurs des passages en question prennent leurs distances face aux pratiques sacrificielles. Il s'agit par conséquent d'un récit didactique expliquant que le seul sacrifice humain demandé par Elohim s'inscrivait dans un contexte tout à fait spécifique (la mise à l'épreuve de l'ancêtre d'Israël), et que Yahvé lui-même a substitué à une telle offrande le sacrifice d'un animal. Gn 22 peut alors se lire comme une polémique très fine contre la pratique des sacrifices humains ${ }^{55}$.

Le récit du sacrifice de la fille de Jephté (Jg 11,29-40) s'inscrit dans une visée comparable. Contrairement à une opinion répandue, il s'agit d'un texte récent datant du IVe siècle avant notre ère. L'auteur commaît à la fois Gn 22 et le mythe d'Iphigénie tel qu il est utilisé par Euripide ${ }^{56}$. Apparemment, l'auteur de Jg 11 veut présenter la fille de Jephté comme une sorte d'Iphigénie hébraïque. Ceci peut s'expliquer par l'influence croissante de la culture hellénistique sur le judaïsme à partir du Ve siècle av. J.-C.

En Jg 11, le sacrifice humain est décliné sur le mode de la tragédie. Dieu n'exige pas le sacrifice humain promis par Jephté, mais il n'intervient pas non plus pour lempêcher.

3 On connaît le rejet des sacrifices aninaliers, et à plus forte raison des sacrifices humains, par le zorosastrisme. Il semble que limportance croissante des sacrifices d'encens en Judée à l'époque perse peut sexpliquer sur cet arrière-fonds, cf. J. Briend. "Malachie 1,11 et l'universalisme", in R. Kuntzmann (éd.), Ce Dieu qui rient. Mélanges offerts à Bernard Renaud. LD 159 (Paris 1995) 191-204: p. 202.

\$2 On peut comprendre Es 66.3 en ce sens. En Ne 10,37 le peuple s'engage à apporter à Y'ahví les premiers-nés de ses fils. Ce texte présuppose pourtant le rachat de ceux-ci, cf. J. Van Seters. loc. cil. (11. 20).

is Cf. supra.

is Cf. P. Maiberger. a Cenesis 22 und die Problematik des Menschenopfers in lsrael», Bibul und Kirche 41 (1986) 10t-112: H.-P. Müller. \& Cenesis 22 und das nulk-Opfer - Erinncrung an einen religionsgeschichtlichev Tatbestand *. BZ +1/2 (1997) 229-2+6.

$\therefore$ Le passage de Elohim à Y'ahsé dans ce récit y contribue peut-être. Voulait-on suggérer que lc Dieu qui demande des sacrifices humains n'est pas le « vrai l'ahvé $\$$ ?

so Pour plus de détails of. T.C. Römer. \& Why Would the Deuteronomists Tell about the Sacrifice of Jejhtahis Daughter? s. JSOT ?? (1998) 27-38. 
De la sorte, nous nous trouvons confrontés à un certain scepticisme théologique à l'égard de l'idée que les diverses actions cultuelles de l'homme (vœu et sacrifice) puissent garantir un contact avec Dieu. L'autcur prend également ses distances en ce qui concerne l'idée d'une pédagogie divine, idée qui était encore sous-jacente dans Cn 22. Il dépeint un Dieu qui se tait face aux aberrations des humains, et qui confronte les hommes avec leur propre cruauté.

À l'aide de ces récits le judaïsme officiel a voulu éradiquer les sacrifices d'enfants; mais peut-être serait-il plus exact de dire qu'il a cherché à fournir une sublimation à ces pratiques. Selon Levenson, «the impulse to sacrifice the first-born son remained potent long after the literal practice had become odious and fallen into desuetude ${ }^{57}$. L'importance de l'Aqeda dans le judaïsme et sa lecture sacrificielle appliqué au peuple juive, de même que l'idée chrétienne du sacrifice du Fils de Dieu, semblent donner raison à cette idée ${ }^{58}$. Mais nous laissons le traitement de ce sujet à des auteurs plus compétents.

\section{Summary}

Die Autoren der hebräischen Bibel stehen dem Menschenopfer kritisch gegenüber Diese Kritik beweist auf ihre Art, daß das Menschenopfer zu biblischer Zeit in Israel und Juda praktiziert wurde. Drei Kategorien sind zu unterscheiden: der herem (ein Kriegsopfer), das Erstlingsopfer und das sogenannte Molekopfer. Die Identität des Molek ist umstritten. Der Autor vertritt die These, daß Molek eine Verballhornung von melek (König) ist; dies war ein Titel für Yahweh, den Nationalgott Israels.

57 J.D. Levenson, op. cit., (n. 24) 52.

${ }^{58} \mathrm{Cf}$. à ce sujet M. Balibutsa, Les sacrifices humains antiques et le mythe christologique, (Kigali 1983). 